\title{
Investigation of Phonon Bottleneck effect on QD lasers
}

\author{
${ }^{a}$ Yi-Shin Su, ${ }^{a}$ Yi Ho, ${ }^{a}$ Wei-Che Chang,,${ }^{\text {a b }}$ Ching-Fuh Lin \\ ${ }^{a}$ with Graduate Inst. of Electron-Optical Eng., National Taiwan Univ., Taiwan \\ ${ }^{b}$ with Dept. of Electrical Eng. and Graduate Inst. of Electronics Eng., National Taiwan Univ., \\ Taiwan
}

\begin{abstract}
The effects of phonon bottleneck effect on the performance of QD laser devices are studied using the rate equation model. Due to phonon bottleneck effect, simultaneous laser oscillations of ground state and excited state can occur. By comparing experimental data and theoretical prediction, the estimated value of intra-dot relaxation time is more than 100ps.
\end{abstract}

Keywords: laser diode, quantum dot, phonon bottleneck effect

\section{INTRODUCTION}

Quantum dot (QD) lasers have the advantage of high differential gain, low threshold current and improved efficiency $^{1}$ because they have the unique delta-function like density of states. Due to high differential gain, quantum dot lasers are expected to have higher modulation response compared with quantum well lasers ${ }^{2}$. However, experimental observation indicates that the modulation speed of quantum dot lasers is lower than that of quantum well lasers ${ }^{3}$. A widely accepted explanation for this behavior is called phonon bottleneck effect. In a quantum well structure, the energy states exist as sub-bands, as shown at the left-hand part of Figure 1. A carrier in the 2nd quantized state can emit or absorb a phonon and jump to states in the 1st quantized subband. By interchange energy and momentum with phonon, an electron in the 2nd quantized subband can fall to the bottom of 1 st quantized subband in about $1 \mathrm{ps}^{4,5,6}$. Compared with electrons, phonons have large momentum and small energy. The initial and final states of carrier transition must have small energy difference and large momentum difference to make momentum and energy conserve for phonon emission or absorption. With 3-dimentional confinement, the quantum dot has only discrete states, right-hand side of Figure 1. The energy separation of these states is much larger than the energy of phonons. Thus, interaction with single phonon is inhibited due to energy non-conservation. Only relaxations through multi-phonon process and carrier-carrier scattering are possible ${ }^{7,89}$. As a result, the intradot relaxation time of carriers in quantum dots is increased. Detailed differential transmission pump probe measurement performed on InGaAs quantum dots shows that the carrier relaxation time from excited state to ground state is on the order of 100ps $200 \mathrm{ps}$ in non-geminately captured dots due to phonon-bottle neck effect, and $6 \mathrm{ps}$ in geminately captured dots due to electron-hole scattering ${ }^{10-12}$. Experiments performed on InAs quantum dots also indicate a 40ps hole intra-dot relaxation time under low excitation density ${ }^{13-15}$.

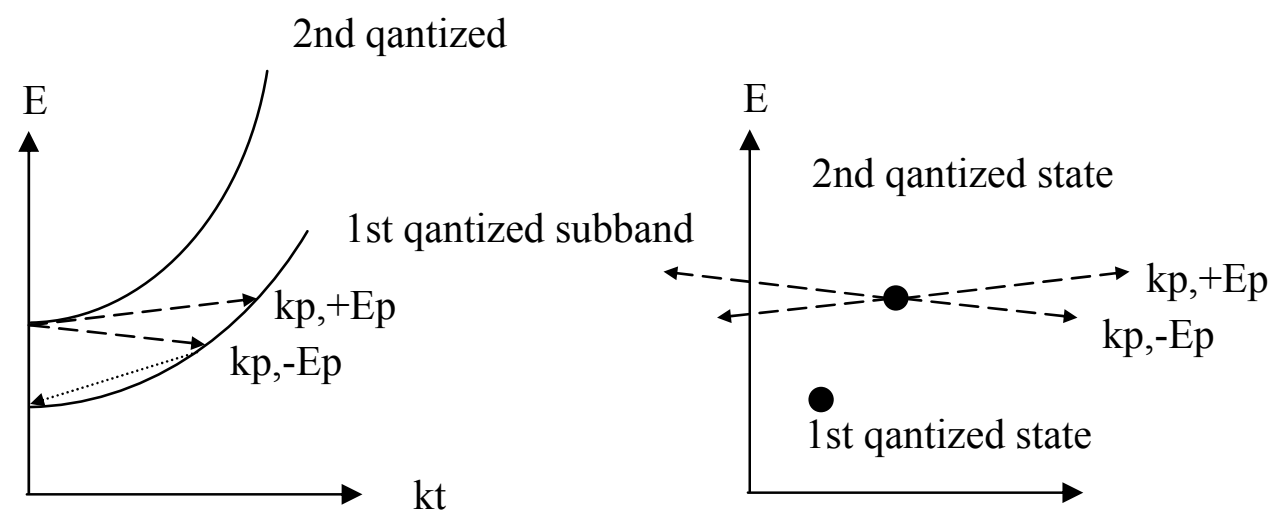

Figure 1: The band structure of quantum well and quantum dot.

Physics and Simulation of Optoelectronic Devices XIV, edited by Marek Osinski, Fritz Henneberger, Yasuhiko Arakawa, Proc. of SPIE Vol. 6115, 61151G, (2006) · 0277-786X/06/\$15 · doi: 10.1117/12.645033 
In this paper, the effects of phonon bottleneck effect on the performance of QD laser devices are studied using the rate equation model. Compared with QW devices, phonon bottleneck effect reduces the gain competition between ground state emission and excited state emission. Thus, simultaneous laser oscillations of ground state and excited state can be observed. According to the investigation, the estimated value of intra-dot relaxation time could be above 150 ps.

\section{THEORY}

To estimate the effect of phonon bottleneck on laser performance, carriers populating ground states and excited states must be treated separately. The following figure illustrates the effects included in our rate equation model. In this model, the injected carriers will fall to the excited state first. Carriers in the excited states have three possible ways to dissipate. They are non-radiative recombination, radiative recombination and relaxation into ground states. The only source of carriers, which occupy the ground states, are carriers falling from the excited states. After falling into ground state, carrier can under go non-radiative recombination or radiative recombination. In this model, we also include the effect of laser emissions contributed by second quantize states. The existence of laser emissions belonging to the excited state will pinch the carrier population of excited state at the threshold level. Thus, the amount of carriers falling from excited states to ground states will also be pinched. The phonon bottleneck effect will affect the carrier relaxation rate. Thus, the amount of carriers falling into ground state will decrease and the power of laser emission of ground states will decrease. However, the current required to drive the excited state to threshold level will decrease, too.

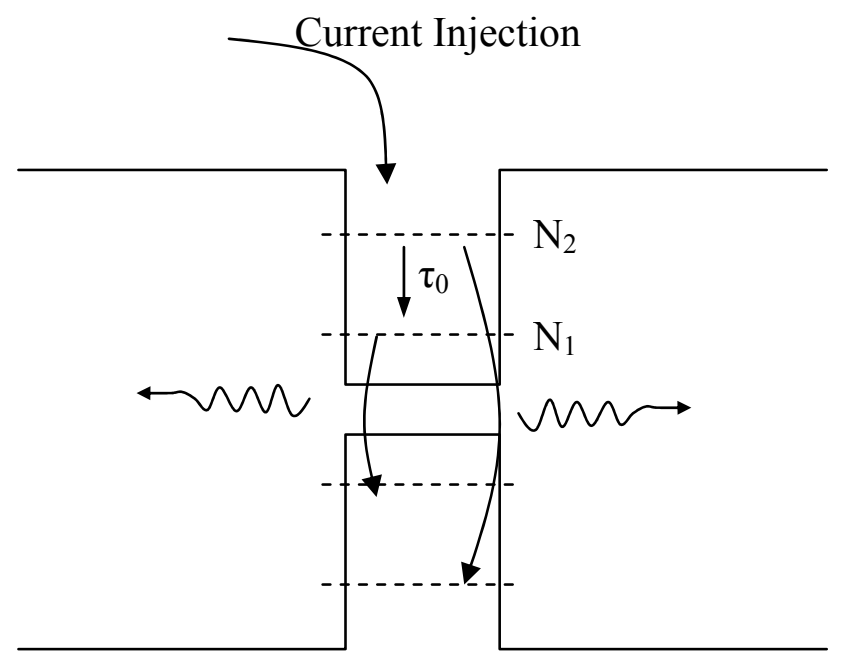

Figure 2: The path of carrier transition modeled.

Following is the rate equation model used for studying phonon bottleneck effect. The first equation models the dynamics of carriers at the excited state. The first term at the right side is for spontaneous recombination. The second term is the rate of carriers falling from excited states down to ground states. The third term is the stimulated emission, and $\mathrm{S}_{2}$ means the photon density of lights emitted by excited state. The final term is carriers contributed by current injection.

The second equation is for carriers in the ground state. The first term at the right side is the spontaneous recombination. The second term is the rate of carriers falling from excited state. The third term is the stimulated 
emission, and $\mathrm{S}_{1}$ means the photon density of lights emitted by the ground state. The last two equations describe the stimulated emission and cavity loss of photons for excited state emission and ground state emission, just like rate equations for conventional diode lasers.

$$
\begin{aligned}
& \frac{d N_{2}}{d t}=-\frac{N_{2}}{\tau_{2}}-\frac{N_{2}}{\tau_{0}}-v_{g} a_{2}\left(N_{2}-N_{t r 2}\right) S_{2}+\frac{\eta_{i} I}{q V} \\
& \frac{d N_{1}}{d t}=-\frac{N_{1}}{\tau_{1}}+\frac{N_{2}}{\tau_{0}}-v_{g} a_{1}\left(N_{1}-N_{t r 1}\right) S_{1} \\
& \frac{d S_{2}}{d t}=-\frac{S_{2}}{\tau_{p 2}}+\Gamma_{2} v_{g} a_{2}\left(N_{2}-N_{t r 2}\right) S_{2}+R_{s p 2} \\
& \frac{d S_{1}}{d t}=-\frac{S_{1}}{\tau_{p 1}}+\Gamma_{1} v_{g} a_{1}\left(N_{1}-N_{t r 1}\right) S_{1}+R_{s p 1}
\end{aligned}
$$

$\tau_{0}:$ inter-band relaxation time

$\mathrm{N}_{1}, \mathrm{~N}_{2}$ : the carrier density of the 1 st and 2nd quantized state

$\mathrm{S}_{1}, \mathrm{~S}_{2}$ : the photon density of transition from the 1 st and 2nd quantized state

I: injection current

$\eta_{\mathrm{i}}$ : internal quantum efficiency

$\mathrm{q}$ : unit electron charge

$\mathrm{V}$ : volume that I is injected through

$\mathrm{v}_{\mathrm{g}}$ : group velocity

$a_{1}, a_{2}$ : differential gain of the 1 st and 2 nd quantized state

$\mathrm{N}_{\mathrm{tr} 1}, \mathrm{~N}_{\mathrm{tr} 2}$ : the transparency level of the 1st and 2nd quantized state

$\tau_{1}, \tau_{2}$ : carrier lifetime of the 1 st and 2 nd quantized state

$\tau_{\mathrm{p} 1}, \tau_{\mathrm{p} 2}$ : photon lifetime of the 1 st and 2 nd quantized state

$\Gamma_{1}, \Gamma_{2}:$ carrier confinement factor

$\mathrm{R}_{\mathrm{sp} 1}, \mathrm{R}_{\mathrm{sp} 2}:$ spontaneous emission rate of the 1 st and 2 nd quantized state

In the simulation, we focus on the effect of intra-dot carrier relaxation time ' $\tau_{0}$ ' and keep others parameters as constants. The spontaneous recombination lifetime of ground state carriers ' $\tau_{1}$ ' is kept at $2 \mathrm{~ns}$. The spontaneous recombination lifetime of excited state carriers ' $\tau_{2}$ ' is also kept at 2 ns. The differential gain of ground state ' $\mathrm{a}_{1}$ ' is $1 \times 10^{-16} \mathrm{~cm}^{2}$. The differential gain of excited state ' $\mathrm{a}_{2}$ ' is $2.7 \times 10^{-16} \mathrm{~cm}^{2}$. The transparency level of both excited states and ground states, ' $\mathrm{N}_{\mathrm{tr} 1}$ ' and ' $\mathrm{N}_{\mathrm{tr} 2}$ ', are set to $1.8 \times 10^{18} \mathrm{~cm}^{-3}$. The photon life times of ground state and excited state emission ' $\tau_{\mathrm{p} 1}$ ' and ' $\tau_{\mathrm{p} 2}$ ' are set to $3 \mathrm{ps}$. The spontaneous emission rates, ' $\mathrm{R}_{\mathrm{sp} 1}$ ' and ' $\mathrm{R}_{\mathrm{sp} 2}$ ', are $1 \times 10^{19} \mathrm{~cm}^{-3} / \mathrm{s}$. The internal quantum efficiency ' $\eta$ ' is 0.8 . Confinement factors ' $\Gamma$ ' and ' $\Gamma_{2}$ ' are 0.01 . The waveguide length is $500 \mu \mathrm{m}$. The waveguide width is $3 \mu \mathrm{m}$. The active layer thickness is $7 \mathrm{~nm}$. Thus, the active layer volume ' $\mathrm{V}$ ' is $7 \mathrm{~nm} \times 500 \mu \mathrm{m} \times 3 \mu \mathrm{m}^{16}$.

Figure $3 \sim$ Figure 6 are the simulated output power vs. injection current curves and carrier population vs. injection current curves. When phonon bottleneck effect is small, carriers can relax from excited states to ground state rapidly. The carrier relaxation time ' $\tau_{0}$ ' is small. Under this condition, very large pumping density is required to increase the carrier population of second quantized states to threshold level. Carriers at the excited states will undergo rapid intra-dot relaxation and fall into ground states. As shown in Figure 3, the threshold pumping density of excited state emission is $8.7 \mathrm{kA} / \mathrm{cm}^{2}$ and the threshold pumping density of ground state emission is $0.43 \mathrm{kA} / \mathrm{cm}^{2}$. The threshold current density of excited states is larger by 20 times, when $\tau 0$ is $100 \mathrm{ps}$. With increasing intra-dot relaxation time, the threshold pumping density of ground state emission increased to $0.5176 \mathrm{kA} / \mathrm{cm}^{2}$, when $\tau 0$ is $500 \mathrm{ps}$, and $0.6211 \mathrm{kA} / \mathrm{cm}^{2}$ when $\tau 0$ is $1000 \mathrm{ps}$. The threshold pumping density for excited state emission decrease from $3.17 \mathrm{kA} / \mathrm{cm}^{2}$ to $2.0703 \mathrm{kA} / \mathrm{cm}^{2}$ when the intra-dot relaxation time increases 
from $100 \mathrm{ps}$ to $1000 \mathrm{ps}$. The threshold current density for excited states is only larger by 3.3 times, when $\tau 0$ is 1000 ps.

Because all injected carriers will fall to excited state first, the slope efficiency of excited state emission does not change with intra-dot relaxation time. Its value is $11.92 \mathrm{~mW}$ per $1 \mathrm{kA} / \mathrm{cm}^{2}$. The slope efficiency of ground state emission decreases with increasing intra-dot relaxation time. With increasing intra-dot relaxation time, less carriers fall into the ground states. Thus, more injected carriers are consumed in the excited states. For lasing corresponding to the ground state, the simulated slope efficiency is $11.3 \mathrm{~mW}$ per $1 \mathrm{kA} / \mathrm{cm}^{2}$ when intra-dot relaxation time is $100 \mathrm{ps}$. This value decreases to 7.94 per $1 \mathrm{kA} / \mathrm{cm}^{2}$, when intra-dot relaxation time is $1000 \mathrm{ps}$.
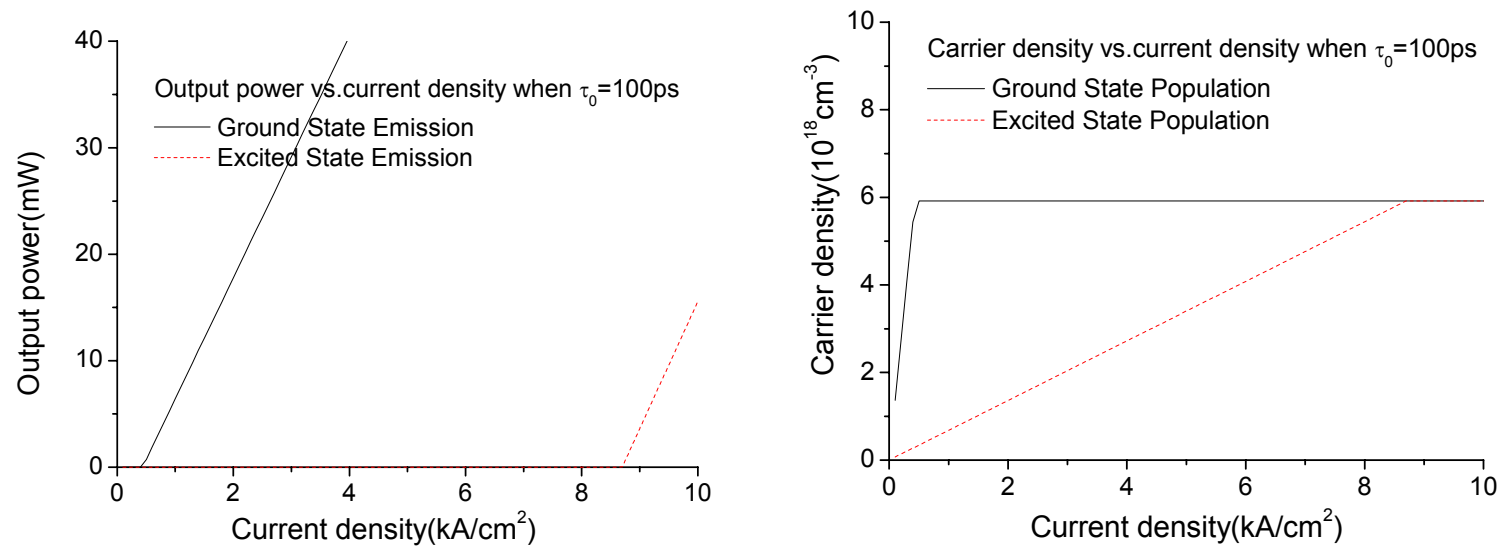

Figure 3: Output power and carrier density vs. J when $\tau_{0} 100 \mathrm{ps}$.
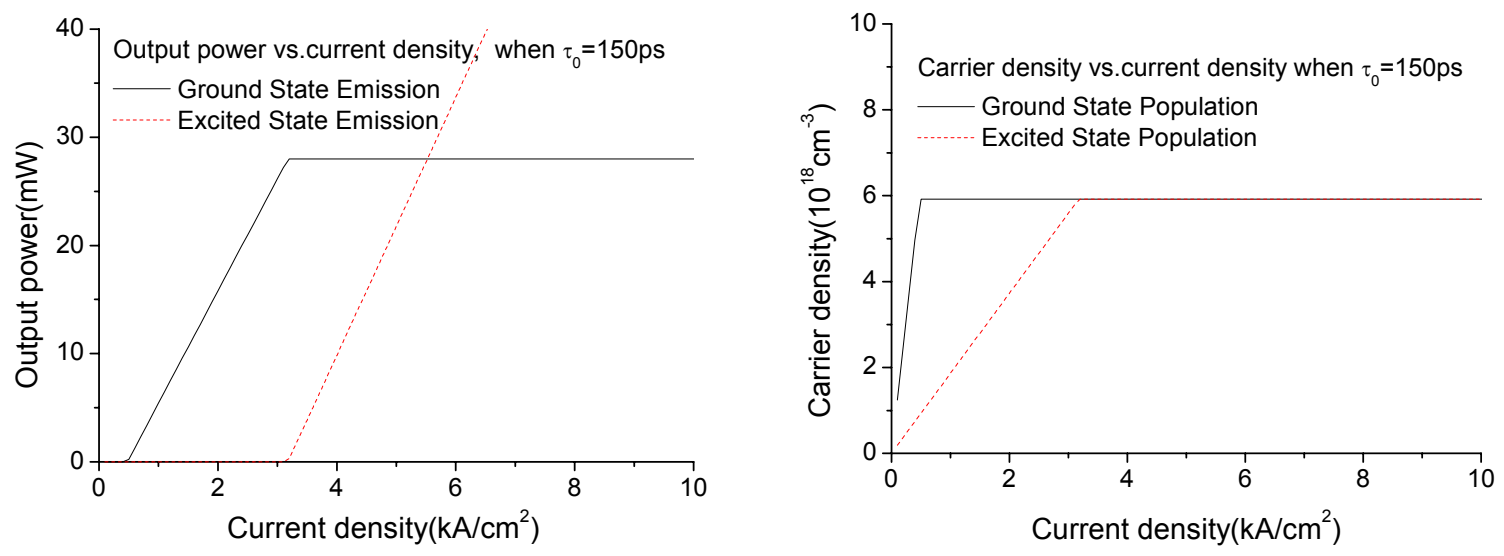

Figure 4: Output power and carrier density vs. J when $\tau_{0} 150 \mathrm{ps}$. 

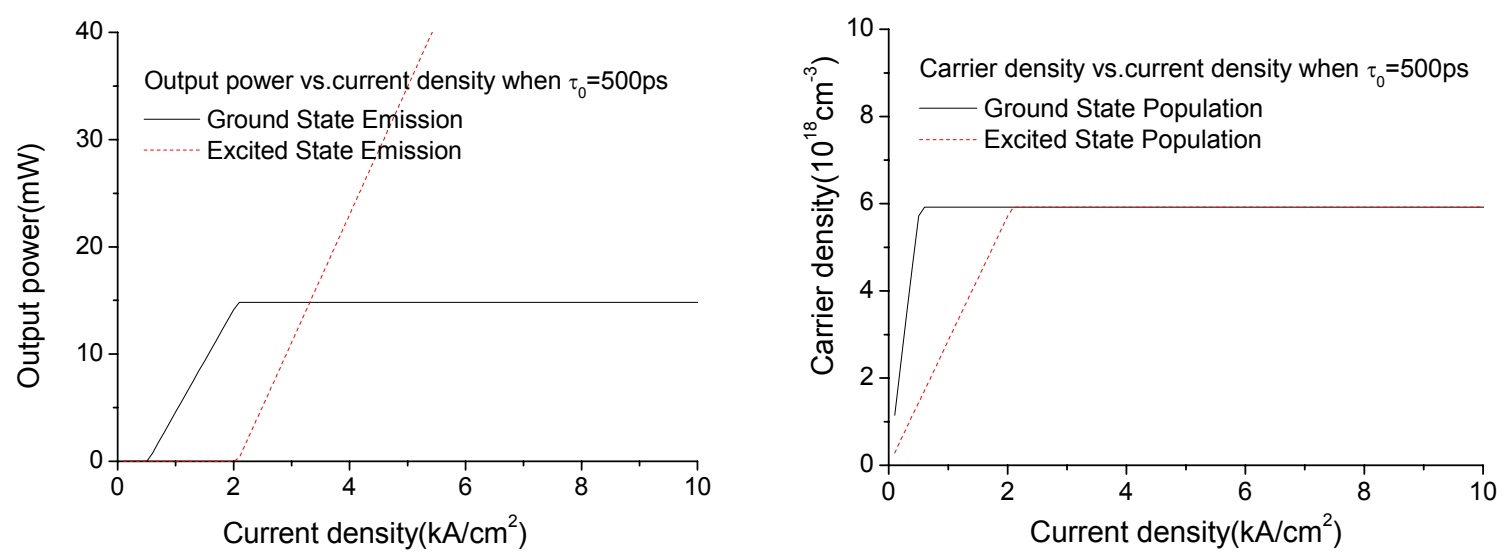

Figure 5: Output power and carrier density vs. J when $\tau_{0} 500$ ps.
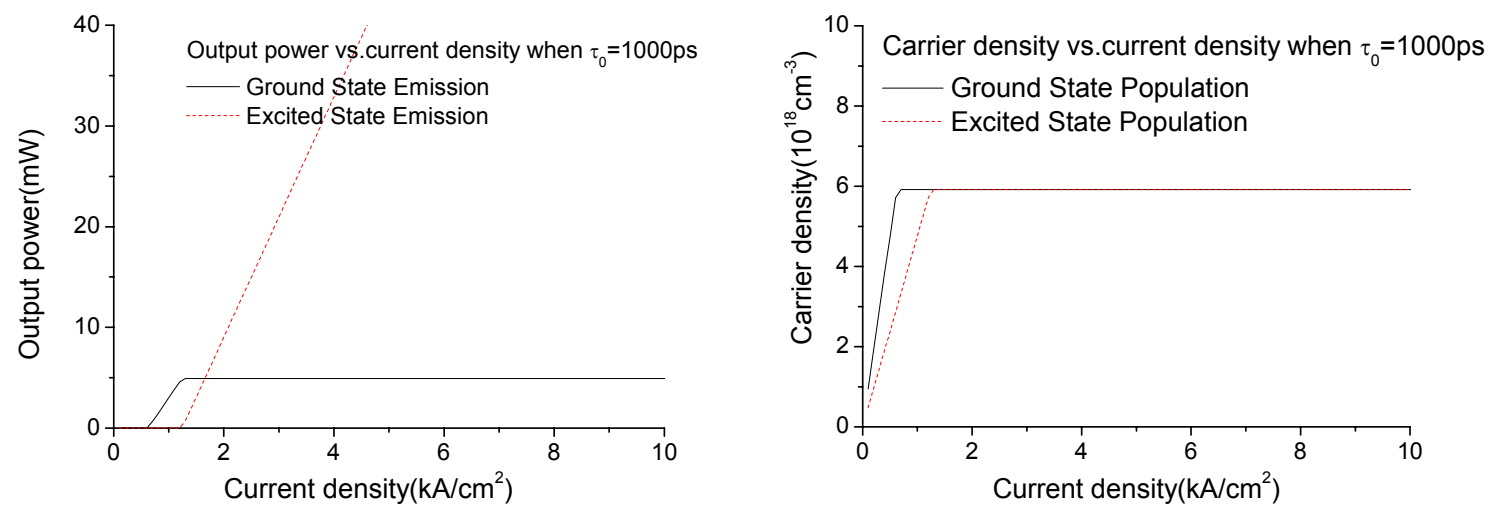

Figure 6: Output power and carrier density vs. J when $\tau_{0} 1000 \mathrm{ps}$.

\section{DEVICE AND EXPERIMENT}

The devices used in this experiment have InAs QDs as the gain material and AlGaAs as current injection layers. The InAs QDs are embedded in InGaAs/GaAs quantum wells for better carrier confinement. As shown in Table 1, two types of QD layers are combined in the active region. The emission wavelength of each type of the QD layer is designed at $1.24 \mu \mathrm{m}$ and $1.28 \mu \mathrm{m}$ respectively. Due to the size dispersion of the quantum dots, the actual emission wavelength covers a large spectral range. Figure 7 is the EL spectrum of a tilted waveguide device fabricated using this wafer. Under $80 \mathrm{~mA}$ injection current, three groups of emission can be identified on the spectrum. The emission contributed by the first quantized states from each QDs is centered around $1260 \mathrm{~nm}$. The emission contributed by the second quantized states from each QD group is centered at $1160 \mathrm{~nm}$. In addition, the emission contributed by higher excited states is revealed in the measured spectrum at $1090 \mathrm{~nm}$. 
Table 1 Wafer structure

\begin{tabular}{|c|c|c|c|c|c|c|c|}
\hline Material & Group & Repeat & $\begin{array}{r}\text { Mole } \\
\text { Fraction(x) }\end{array}$ & $\begin{array}{r}\text { Thickness } \\
\text { (nm) }\end{array}$ & Doping & Type & Dopant \\
\hline GaAs & & & & 100 & $5 \mathrm{e} 19$ & $\mathrm{P}$ & $\mathrm{C}$ \\
\hline $\mathrm{Al}(\mathrm{x}) \mathrm{Ga}(1-\mathrm{x}) \mathrm{As}$ & & & $0.35 \sim 0$ & 20 & $3 \mathrm{e} 18$ & $\mathrm{P}$ & $\mathrm{C}$ \\
\hline $\mathrm{Al}(\mathrm{x}) \mathrm{Ga}(1-\mathrm{x}) \mathrm{As}$ & & & 0.35 & 700 & $1 \mathrm{e} 18$ & $\overline{\mathrm{P}}$ & $\mathrm{C}$ \\
\hline $\operatorname{Al}(\mathrm{x}) \mathrm{Ga}(1-\mathrm{x}) \mathrm{As}$ & & & 0.35 & 500 & $5 \mathrm{e} 17$ & $\overline{\mathrm{P}}$ & $\mathrm{C}$ \\
\hline GaAs & 2 & 5 & & 35 & & $\mathrm{U} / \mathrm{D}$ & \\
\hline $\operatorname{In}(x) \operatorname{Ga}(1-x) A s$ & 2 & 5 & 0.15 & 5 & & $\mathrm{U} / \mathrm{D}$ & \\
\hline InAs(for 1.24um ) & 2 & 5 & & 0.7 & & $\mathrm{U} / \mathrm{D}$ & \\
\hline GaAs & 2 & 5 & & 35 & & $\mathrm{U} / \mathrm{D}$ & \\
\hline $\operatorname{In}(x) \operatorname{Ga}(1-x) A s$ & 2 & 5 & 0.15 & 5 & & $\mathrm{U} / \mathrm{D}$ & \\
\hline $\operatorname{InAs}($ for $1.28 \mathrm{um})$ & 2 & 5 & & 0.8 & & $\mathrm{U} / \mathrm{D}$ & \\
\hline GaAs & & & & 35 & & $\mathrm{U} / \mathrm{D}$ & \\
\hline $\mathrm{Al}(\mathrm{x}) \mathrm{Ga}(1-\mathrm{x}) \mathrm{As}$ & & & 0.35 & 500 & $5 \mathrm{e} 17$ & $\mathrm{~N}$ & $\mathrm{Si}$ \\
\hline $\mathrm{Al}(\mathrm{x}) \mathrm{Ga}(1-\mathrm{x}) \mathrm{As}$ & & & 0.35 & 1000 & $1 \mathrm{e} 18$ & $\mathrm{~N}$ & $\mathrm{Si}$ \\
\hline $\mathrm{Al}(\mathrm{x}) \mathrm{Ga}(1-\mathrm{x}) \mathrm{As}$ & & & $0 \sim 0.35$ & 20 & $3 \mathrm{e} 18$ & $\mathrm{~N}$ & $\mathrm{Si}$ \\
\hline GaAs & & & & 500 & $3 \mathrm{e} 18$ & $\mathrm{~N}$ & $\mathrm{Si}$ \\
\hline GaAs substrate & $\mathrm{N}+\mathrm{Ga}$ & s 3 inch & & & & & \\
\hline
\end{tabular}

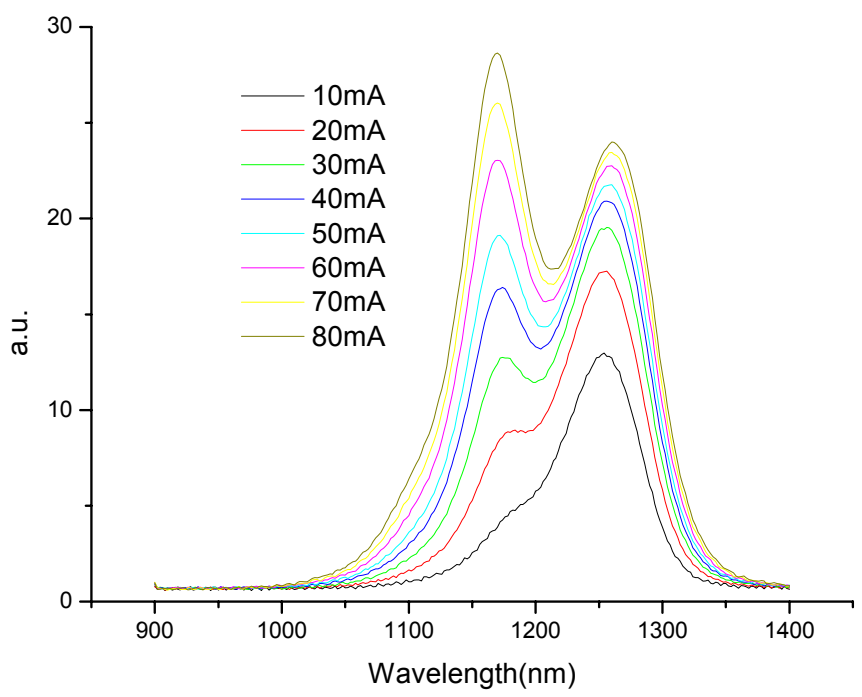

Figure 7: EL emission of a $500 \mu \mathrm{m}$ tilt waveguide device by this wafer. 
After MOCVD growth of epi-structure, two section laser diode devices are fabricated. The process flow consists of waveguide etching, passivation dielectric growth, $\mathrm{p}$-contact metallization, lapping, and $\mathrm{n}$-contact metallization. These devices use double channel waveguide. The channels are etched by $\mathrm{HBr}: \mathrm{H}_{2} \mathrm{O}_{2}: \mathrm{HCl}_{2} \mathrm{H}_{2} \mathrm{O}$ solution, the etching mask is AZ5214E photoresist. The etching mask of waveguide is $2.3 \mu \mathrm{m}$ in width. The etched ridge waveguide bottom is $4 \mu \mathrm{m}$ in width. The etched depth is $100 \mathrm{~nm} \sim 200 \mathrm{~nm}$ above the undoped GaAs barrier. The passivation dielectric is silicon dioxide deposited by PECVD under $300^{\circ} \mathrm{C}$. The oxide on top of waveguide is removed for electrical contact. Due to the small dimension of waveguide top, a self-aligned procedure is developed to define the region for oxide removal. The electrical contact on p-type GaAs is Ti/Pt/Au $1 \mathrm{kA} / 1 \mathrm{kA} / 3 \mathrm{kA}$ followed by rapid thermal annealing. After p-contact metallization, the wafer is lapped down to $100 \mu \mathrm{m}$. AuGe/Ni/Au is applied as contact on $\mathrm{n}-\mathrm{GaAs}$ substrate. Following is the process flow chart.

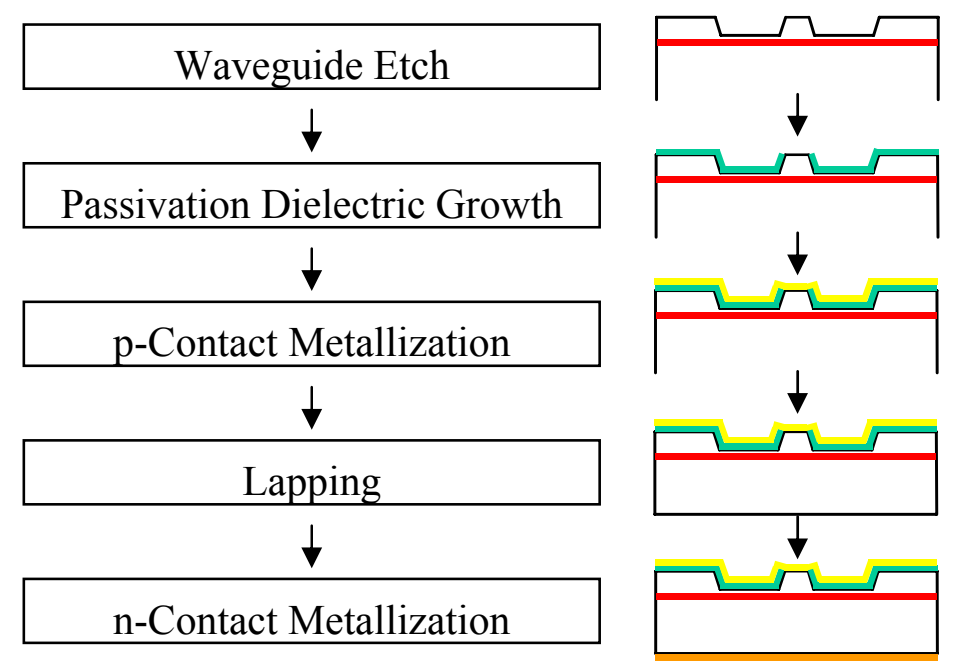

Figure 8: Process flow Chart

With total 500um cavity length, the laser diode has $21 \mathrm{~mA}$ threshold current (Figure 9). For current below 60mA, the laser output has the emissions corresponding to only the ground state (Figure 10). When we further increase the injection current, lasing corresponding to the excited state is observed. The corresponding threshold current of the excited state emission is at $66.79 \mathrm{~mA}$. The threshold current of excited state emission is 3.18 times that of the ground state transition. As shown in the L-I curve, the slope efficiency of the ground state emission decreased dramatically as the excited state emission reaches threshold. As the lasing at the excited state occurs, because the carrier population of the excited state is pinched at threshold level, the amount of carriers falling into ground states is also fixed. The slope efficiency of the ground state emission is only 0.56 times that of the excited state emission, because some of the injected carriers are wasted due to some the nonradiative recombination of carriers in the excited states. Compared with the theoretical result, the intra-dot relaxation time should be close to 500ps to match the ratio of threshold currents between the ground state and the excited state. However, the intra-dot relaxation time should be close to $1000 \mathrm{ps}$ to match the ratio of slope efficiencies between the ground state and the excited state. 


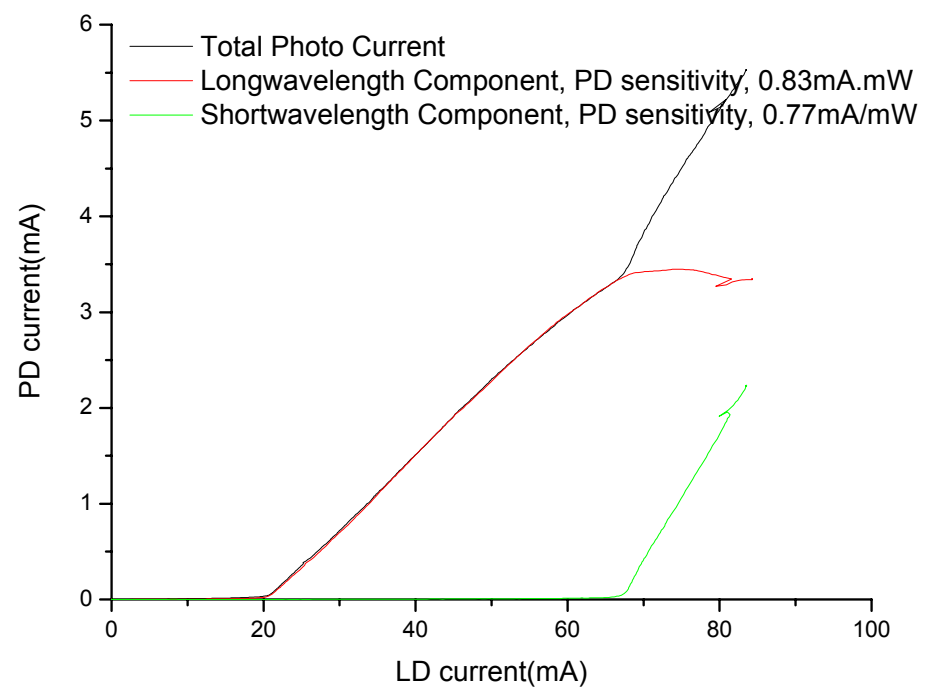

Figure 9: L-I curve of the device

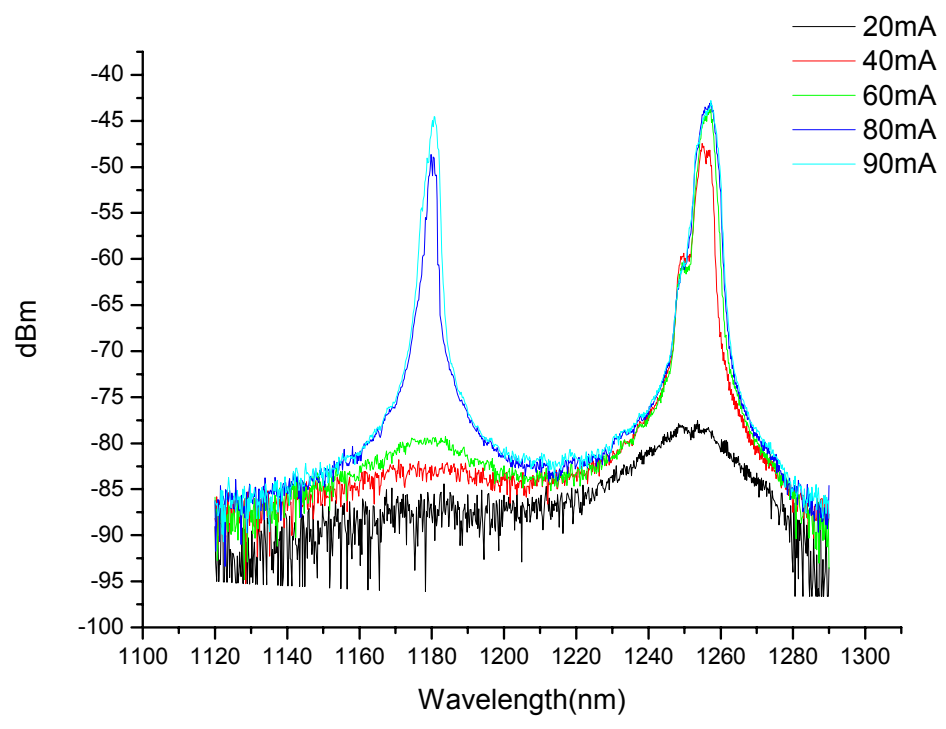

Figure 10: Emission Spectrum of the device 


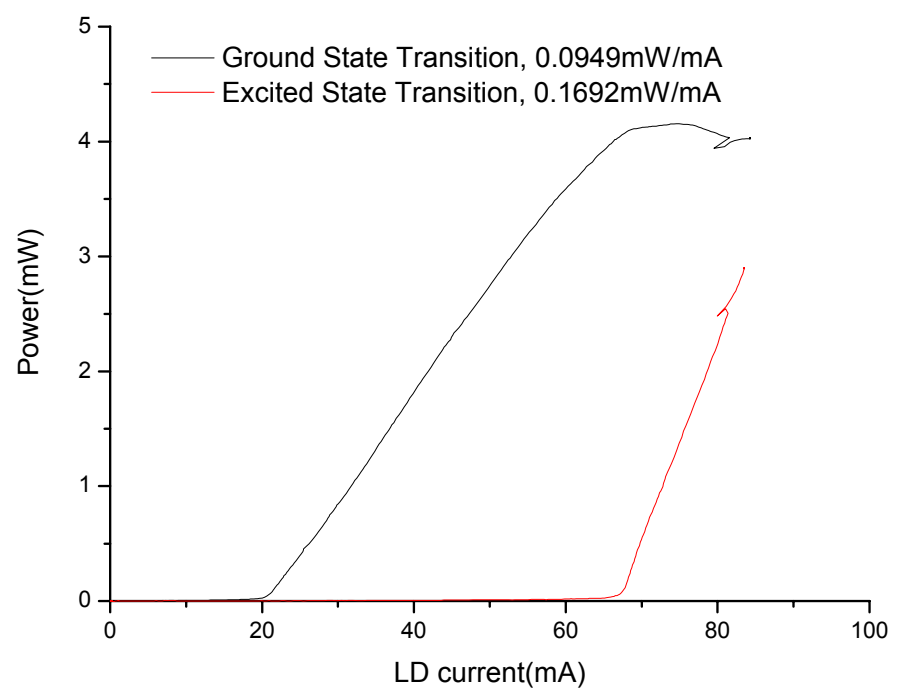

Figure 11: L-I curve of each wavelength

\section{CONCLUSION}

The effects of phonon bottleneck effect on the performance of QD devices are studied numerically using the rate equation model. Strong phonon bottleneck effect results in simultaneous laser oscillations of 1st and 2nd quantized states of a QD laser. In QW devices, the excess energy of carrier relaxation can be released to other carriers, such as Auger effect, or dissipated as phonons. Thus, strong competition between laser oscillations of 1st and 2nd quantized states will prevent simultaneous laser oscillation. In the case of QDs, the two processes are forbidden because the momentum is not conserved. For an electron to relax from $2^{\text {nd }}$ quantized state to $1^{\text {st }}$ quantized state, excess energy can only be transferred to carriers outside the QD by coulomb interaction Thus, it takes more time for carriers to relax into first quantized state in QD structures.

According to the rate equation model, the carrier relaxation time between 1st and 2nd quantized state must be more than $100 \mathrm{ps}$ for simultaneous laser oscillation to occur. This value is far more than the carrier relaxation time of a QW structure. Thus, the existence of phonon bottleneck effect can be confirmed by the observation of two-mode laser oscillation of a QD laser.

\section{Acknowledgement}

The authors wish to acknowledge support of National Science Council under contract number NSC 94-2120-M-002-010 and NSC 94-2112-M-002-009.

\section{REFERENCE}

1. Q. Xie, P. Chen, A. Kalburge, T. R. Ramachandran, A. Nayfonov, A. Konkar, and A. Madhukar, "Realization of optically active strained InAs island quantum boxes on GaAs (100) via molecular beam epitaxy and the role of island induced strain fields," J. Cryst. Growth, vol. 150, p. 357, 1995.

2. L.A. Coldren and S. W. Corzine, "Diode Lasers and Photonic Integrated Circuits", pp199-207, Willey, New 
York, 1995

3. D. Klotzkin and P. Bhattacharya, "Temperature dependence of dynamic and DC characteristics of quantum well and quantum-dot lasers: A comparative study," J. Lightwave Technol., vol. 17, pp. 1634-1642, 1999.

4. J. Faist, F. Capasso, D. L. Sivco, C. Sirtori, A. L. Hutchinson, and A. Y. Cho, "Quantum cascade laser," Science, vol. 264, pp. 553-556, 1994.

5. J. Faist, F. Capasso, C. Sirtori, D. L. Sivco, A. L. Hutchinson, and A. Y. Cho, "Vertical transition quantum cascade laser with Bragg confined excited state," Appl. Phys. Lett., vol. 66, pp. 538-540, 1995.

6. U. Bockelmann and G. Bastard, "Phonon scattering and energy relaxation in two-, one-, and zero-dimensional electron gases," Phys. Rev. B, vol. 42, pp. 8947-8951, 1990.

7. A. V. Uskov, I. Magnusdottir, B. Tromborg, J. Mørk, and R. Lang, "Line broadening caused by coulomb carrier - carrier correlations and dynamics of carrier capture and emission in quantum dots," Appl. Phys. Lett., vol. 79, p. 1679, 2001.

8. R. Heitz, M. Grundmann, N. N. Ledentsov, L. Eckey, M. Veit, D. Bimberg, V. M. Ustinov, A. Yu. Egorov, A. E. Zhukov, P. S. Kope'v, and Zh. I. Alferov, "Multiphonon-relaxation processes in self-organized InAs/GaAs quantum dots," Appl. Phys. Lett., vol. 68, pp. 361-363, 1996.

9. R. Heitz, A. Kalburge, Q. Xie, M. Grundmann, P. Chen, A. Hoffmann, A. Madhukar, and D. Bimberg, "Excited states and energy relaxation in stacked InAs/GaAs quantum dots," Phys. Rev. B, vol. 57, pp. 9050-9060, 1998.

10. J. Urayama, T. B. Norris, H. Jiang, J. Singh, and P. Bhattacharya, "Temperature-dependent carrier dynamics in self-assembled InGaAs quantum dots," Appl. Phys. Lett., vol. 80, pp. 2162-2164, 2002.

11. K. Kim, J. Urayama, T. B. Norris, J. Singh, J. Phillips, and P. Bhattacharya, "Gain dynamics and ultrafast spectral hole burning in In(Ga)As self-organized quantum dots," Appl. Phys. Lett., vol. 81, pp. 670-672, 2002. 12. J. Urayama, T. Norris, J. Singh, and P. Bhattacharya, "Observation of phonon bottleneck in quantum dot electronic relaxation," Phys. Rev. Lett., vol. 86, pp. 4930-4933, 2001.

13. R. Heitz, H. Born, F. Guffarth, O. Stier, A. Schliwa, A. Hoffmann, and D. Bimberg," Existence of a phonon bottleneck for excitons in quantum dots" Phys. Rev. B, vol. 64, pp., 2001.

14. R. Heitz, M. Veit, N.N. Ledentsov, A. Hoffmann, D. Bimberg, V.M. Ustinov, P.S. Kop'ev, and Zh.I. Alferov, Energy relaxation by multiphonon processes in InAs/GaAs quantum dots", Phys. Rev. B, 56, 10435,1997

15. K.H. Schmidt, G. Medeiros-Ribeiro, M. Oestereich, P.M. Petroff, and G.H. Do"hler, Carrier relaxation and electronic structure in InAs self-assembled quantum dots", Phys. Rev. B, 54, $11346 \sim 1996$ !.

16. P. Bhattacharya, S. Ghosh, S. Pradhan, J. Singh, Z-K. Wu, J. Urayama, K. Kim, and T. Norris, "Carrier dynamics and high-speed modulation properties of tunneling injection InGaAs-GaAs quantum-dot lasers ", IEEE Journal of Quantum Electronics, Vol.39, no.8, pp.952-962, 2003. 